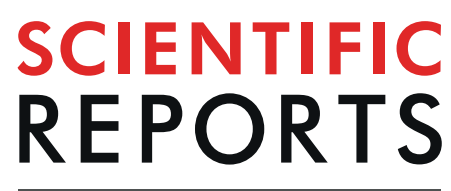

natureresearch

\title{
Complex approach for analysis of snake venom $\alpha$-neurotoxins binding to HAP, the high-affinity peptide
}

Denis S. Kudryavtsev ${ }^{1,11}$, Valentin M. Tabakmakher ${ }^{1,2,11}$, Gleb S. Budylin ${ }^{3,11}$, Natalia S. Egorova ${ }^{1}$, Roman G. Efremov ${ }^{1,4,5}$, Igor A. Ivanov ${ }^{1}$, Svetlana Yu. Belukhina ${ }^{6}$, Artjom V. Jegorov ${ }^{1}$, Igor E. Kasheverov ${ }^{1,7}{ }^{\text {, Elena V. Kryukova }}{ }^{1}$, Irina V. Shelukhina ${ }^{1}$, Evgeny A. Shirshin ${ }^{8,9}$, Nadezhda G. Zhdanova ${ }^{8}$, Maxim N. Zhmak ${ }^{1}$ \& Victor I. Tsetlin ${ }^{1,10^{*}}$

Snake venom $\alpha$-neurotoxins, invaluable pharmacological tools, bind with high affinity to distinct subtypes of nicotinic acetylcholine receptor. The combinatorial high-affinity peptide (HAP), homologous to the C-loop of $\alpha 1$ and $\alpha 7 \mathrm{nAChR}$ subunits, binds biotinylated $\alpha$-bungarotoxin ( $\alpha \mathrm{Bgt})$ with nanomolar affinity and might be a protection against snake-bites. Since there are no data on HAP interaction with other toxins, we checked its binding of $\alpha$-cobratoxin $(\alpha \mathrm{Ctx})$, similar to $\alpha \mathrm{Bgt}$ in action on nAChRs. Using radioiodinated $\alpha$ Bgt, we confirmed a high affinity of HAP for $\alpha$ Bgt, the complex formation is supported by mass spectrometry and gel chromatography, but only weak binding was registered with $\alpha \mathrm{Ctx}$. A combination of protein intrinsic fluorescence measurements with the principal component analysis of the spectra allowed us to measure the HAP- $\alpha$ Bgt binding constant directly ( $29 \mathrm{nM})$. These methods also confirmed weak HAP interaction with $\alpha \mathrm{Ctx}(>10000 \mathrm{nM})$. We attempted to enhance it by modification of HAP structure relying on the known structures of $\alpha$-neurotoxins with various targets and applying molecular dynamics. A series of HAP analogues have been synthesized, HAP[L9E] analogue being considerably more potent than HAP in $\alpha \mathrm{Ctx}$ binding $(7000 \mathrm{nM})$. The proposed combination of experimental and computational approaches appears promising for analysis of various peptide-protein interactions.

$\alpha$-Neurotoxins are snake venom proteins serving as accurate tools in research on nicotinic acetylcholine receptors (nAChRs) (see reviews ${ }^{1-3}$ ). Their valuable feature is the capacity to distinguish certain nAChRs subtypes: short-chain $\alpha$-neurotoxins bind only to the muscle-type nAChRs, while the long ones, such as $\alpha$-bungarotoxin $(\alpha \mathrm{Bgt})$ and $\alpha$-cobratoxin ( $\alpha \mathrm{Ctx}$ ), are blocking muscle-type nAChRs and the neuronal ones containing $\alpha 7, \alpha 9$ and $\alpha 9 / \alpha 10$ subunits $^{4-6}$. The spatial structure of all $\alpha$-neurotoxins consists of three $\beta$-structural loops, giving them the name of "three-finger" proteins (with the abbreviations 3FP or TFPs), their arrangement being maintained by 4 disulfide bridges in the short and by 5 disulfides in the long $\alpha$-neurotoxins. Interestingly, the TFP family embraces not only numerous $\alpha$-neurotoxins, but also diverse snake venom proteins acting specifically on different targets (ion channels, G-protein coupled receptors, enzymes) or, in the case of cytotoxins, just disrupting cell membranes $\left(\mathrm{see}^{7}\right)$. The interest to $\alpha$-neurotoxins in recent years was invigorated by the discovery that some

\footnotetext{
${ }^{1}$ Shemyakin-Ovchinnikov Institute of Bioorganic Chemistry, Russian Academy of Sciences, Moscow, 117997, Russia. ${ }^{2}$ School of Biomedicine, Far Eastern Federal University, Vladivostok, 690950, Russia. ${ }^{3}$ Faculty of Physics, National Research University Higher School of Economics, Moscow, 101000, Russia. ${ }^{4}$ National Research University Higher School of Economics, Moscow, 101000, Russia. ${ }^{5}$ Moscow Institute of Physics and Technology (State University), Dolgoprudny, 141701, Moscow Oblast, Russia. ${ }^{6}$ Faculty of Biotechnology, Moscow State University, Moscow, 119991, Russia. ${ }^{7}$ Sechenov First Moscow State Medical University, Institute of Molecular Medicine, Moscow, 119991, Russia. ${ }^{8}$ Department of Physics, M.V. Lomonosov Moscow State University, Moscow, 119992, Russia. ${ }^{9}$ Institute of spectroscopy of the Russian Academy of Sciences, Troitsk, Moscow, 108840, Russia. ${ }^{10}$ PhysBio of MePhi, 115409, Moscow, Russia. ${ }^{11}$ These authors contributed equally: Denis S. Kudryavtsev, Valentin M. Tabakmakher and Gleb S. Budylin.*email: vits@ibch.ru
} 
so-called Ly6 proteins, which also have a three-finger folding and are present in a wide range of organisms from Drosophila to human beings, interact with nAChRs (see reviews ${ }^{1,8}$ ). Such Ly6 proteins as Lynx1 (attached to the membrane by glycosylphosphatidylinositol anchor) or SLURP1 (secreted protein) are endogenous regulators of the physiological and pathophysiological functions of $\mathrm{nAChRs}$ and design of novel drugs on their basis requires an understanding of mechanisms of their action. Here only first steps were made (see recent references in ${ }^{9}$ ), and we believe that the assistance may come from research on $\alpha$-neurotoxins for which a wealth of information is available, including comprehensive analysis of their interactions with nAChRs (mutations and electrophysiology), as well as the X-ray and NMR structures for $\alpha$-neurotoxin complexes with the nAChR models.

The best $\mathrm{nAChR}$ models are water-soluble acetylcholine-binding proteins (AChBPs). The X-ray structure of AChBP for the first time demonstrated how a ligand-binding domain (LBD) of the nAChR should look ${ }^{10,11}$. Then followed the X-ray structures of AChBP complexes with various agonists and antagonists (see review ${ }^{12}$ ), including those with $\alpha$-neurotoxins and $\alpha$-conotoxins, as well as the $\alpha$ Bgt complexes with the ligand-binding domains of the $\mathrm{nAChR} \alpha 1$ and $\alpha 9$ subunits $^{13,14}$. These structures were utilized to deduce information about the binding sites of distinct whole-size nAChRs.

The role of the nAChR models or "mimics" to a certain extent can be played by relatively short peptides. Indeed, $\alpha$-neurotoxins for a long time were known to bind to synthetic fragments of nAChR $\alpha 1$ and $\alpha 7$ subunits encompassing their C-loops, as well as to the combinatorial peptides sharing a certain similarity with this loop ${ }^{15}$. The affinity of such binding may be sufficiently high, and recently this property was utilized by introducing the respective sequences into the extracellular parts of diverse receptors, differing from the nAChRs. Fluorescent or biotinylated $\alpha$ Bgt derivatives made possible detecting of such receptors in the cells and monitoring receptor assembly and cell internalization; among the successful examples were such ion channels as $\alpha$-amino-3-hydroxy -5-methyl-4-isoxazolepropionic acid (AMPA) receptors, ionotropic $\gamma$-aminobutiryc acid (GABAA) receptors and voltage-gated potassium Kv4.2 channels, as well as such G-protein coupled receptors as $\gamma$-aminobutiryc acid receptors $(\mathrm{GABAB})^{16}$.

Our attention was attracted to a 13-membered peptide HAP ("high affinity peptide"), which binds biotinylated $\alpha$ Bgt with a high affinity $(2-4 \mathrm{nM})$, characteristic for the $\alpha \mathrm{Bgt}$ binding to the whole-size $\mathrm{nAChRs}$. The X-ray structure of HAP in complex with $\alpha$ Bgt and ${ }^{1} \mathrm{H}-\mathrm{NMR}$ structure of a close HAP homolog bound to $\alpha$ Bgt have been solved ${ }^{17,18}$. Such high affinity stimulated the idea that HAP might be used as a remedy against snake bites. The principle possibility of such an approach against poisoning was recently shown by application of AChBP which could bind long, but not short $\alpha$-neurotoxins ${ }^{19,20}$. This line of research is still of interest. Previously, only the interaction of HAP with biotinylated $\alpha B g t$ has been described. One of the purposes of our work was to check how HAP interacts with a different long-chain $\alpha$-neurotoxin, namely $\alpha$-cobratoxin from the Naja kaouthia which binds to nAChRs almost with the same affinity as $\alpha$ Bgt. In competition with radioiodinated $\alpha$ Bgt we confirmed the earlier reported high affinity binding of HAP to biotinylated $\alpha$ Bgt. However, we registered only very weak binding to $\alpha$ Ctx. It prompted us to analyze available data on the spatial structures of $\alpha$ Bgt and $\alpha$ Ctx in complexes with the peptide fragments and nAChR models and to design a HAP analog with the increased affinity for $\alpha \mathrm{Ctx}$. For this purpose we combined computational approaches, radiolgand analysis, gel chromatography, mass spectrometry and fluorescence spectroscopy for a thorough analysis of interactions between HAP and its analogs with $\alpha$ Bgt and $\alpha \mathrm{Ctx}$. Although for $\alpha \mathrm{Ctx}$ we succeeded in only moderate increase in the affinity, we hope that the proposed approach will be helpful for analysis of various peptide-protein interactions.

\section{Materials and methods}

Synthesis of HAP and its analogs. Solid-phase synthesis utilizing the Fmoc strategy was performed to make the peptides. The first amino acid residue was attached to Wang-type polystyrene resin (Iris Biotech, Germany) by symmetric anhydride method. Polypeptide chain assembly was performed on Syro II automatic peptide synthesizer (MultiSynTech AG, Germany) with DIC/HOAt activation.

Preparative purification was carried out as described previously ${ }^{21}$ using a Gilson HPLC system (333/334 pump with 215 liquid handler) equipped with a YMC Triart $10 \mathrm{um} 30 \times 150 \mathrm{~mm}$ column and UV detection at 210 and $280 \mathrm{~nm}$. Peptides elution was achieved by addition of a $\mathrm{H}_{2} \mathrm{O}$-acetonitrile in the $10-55 \%$ gradient with $0.1 \%$ v/v CF3COOH. HPLC-MS analysis was performed using Thermo Finnigan LCQ Deca XP ion trap instrument with Thermo Accela UPLC system equipped with Waters Atlantis T3 $3 \mathrm{um} 150 \times 2 \mathrm{~mm}$ column. Detection was achieved by UV-VIS DAD and full scan MS (ESI+, 150-2000 Th).

Radioligand analysis of the HAP and its analogs interactions with $\alpha \mathrm{Bgt}$ and $\alpha \mathrm{Ctx}$. Competition of HAP and its analogues with mono-iodinated ${ }^{125} \mathrm{I}-\alpha \mathrm{Bgt}$ and ${ }^{125} \mathrm{I}-\alpha \mathrm{Ctx}^{22}(500 \mathrm{Ci} / \mathrm{mmol})$ was carried out on two targets - muscle-type nAChR in membrane preparation of Torpedo californica ray electric organ and neuronal human $\alpha 7 \mathrm{nAChR}$ transfected in GH4C1 cell line. For competitive radioligand assays, suspensions of T. californica $\mathrm{nAChR}$-rich membranes (1.25 $\mathrm{nM} \alpha$-bungarotoxin binding sites, kindly provided by Prof. F. Hucho, Institute for Chemistry and Biochemistry, Freie Universität Berlin, Germany) in $50 \mu \mathrm{L}$ of $20 \mathrm{mM}$ Tris-HCl buffer $\mathrm{pH} 8.0$, containing $1 \mathrm{mg} / \mathrm{ml}$ BSA (binding buffer) or human $\alpha 7 \mathrm{nAChR}$ transfected cells $(0.4 \mathrm{nM} \alpha$-bungarotoxin binding sites, received from Eli Lilly and Company, London, UK) in $50 \mu \mathrm{L}$ of binding buffer were incubated for $3 \mathrm{~h}$ at room temperature with various amounts of HAP or its analogues, followed by an additional 5 min incubation with $0.5-0.9 \mathrm{nM}$ of radioligands. Nonspecific binding was determined by preliminary incubation of the preparations with $30 \mu \mathrm{M} \alpha$-cobratoxin. The membrane and cell suspensions were applied to glass $\mathrm{GF} / \mathrm{C}$ filters (Whatman, Maidstone, UK) presoaked in $0.25 \%$ polyethyleneimine, and unbound radioactivity was removed from the filter by washing $(3 \times 3 \mathrm{ml})$ with $20 \mathrm{mM}$ Tris- $\mathrm{HCl}$ buffer, $\mathrm{pH} 8.0$ containing $0.1 \mathrm{mg} / \mathrm{ml} \mathrm{BSA}$ (washing buffer). The bound radioactivity was determined using a Wizard 1470 Automatic Gamma Counter ${ }^{23}$. 
Computer modeling. The experimentally determined structure (atomic coordinates) of $\alpha$ Bgt complexes with HAP and HAP[L9E] were obtained from the RCSB Protein Data Bank (PDB ID: 1HC9, 1HAJ, respectively $)^{17,18}$. Structure of $\alpha$ Ctx was derived from its complex with $L s$-AChBP (PDB ID: $1 Y 5^{24}$ ). The models of $\alpha$ Ctx complexes with HAP and HAP[L9E] were built on the basis of complexes with $\alpha$ Bgt (PDB ID: 1HC9 and $1 \mathrm{HAJ})$ by 3D alignment $\alpha$ Ctx onto $\alpha$ Bgt and subsequent removal of $\alpha$ Bgt. Models of HAP variants with point amino acid substitutions complexed to $\alpha \mathrm{Ctx}$ and $\alpha \mathrm{Bgt}$ were built using in silico mutagenesis in the PyMOL program (The PyMOL Molecular Graphics System, Version 1.8 Schrödinger, LLC.).

Molecular dynamics simulations. Molecular dynamics (MD) simulations of free HAP, HAP[L9E] and other point mutation variants of HAP peptide, free $\alpha$-neurotoxins, as well as their complexes, were performed in explicitly defined water environment with the GROMACS 5.1.2 software ${ }^{25}$ using the Gromos $9643 \mathrm{a} 3$ parameters set $^{26}$. Peptides' complexes with $\alpha$-neurotoxins were solvated inside $(6.0-6.7 \mathrm{~nm})^{3}$ cubic boxes with 6900-9560 water molecules; the SPC water model ${ }^{27}$ was used; the required number of $\mathrm{Cl}^{-}$or $\mathrm{Na}^{+}$ions to maintain electroneutrality was added. Analogously, free HAP and HAP[L9E] peptides and free $\alpha$-neurotoxins were solvated in $(4.32-4.42 \mathrm{~nm})^{3} /(6.28-6.76 \mathrm{~nm})^{3}$ boxes with $2535-2700 / 7660-9615$ water molecules. Simulations were carried out with a time step of $2 \mathrm{fs}$, imposing 3D periodic boundary conditions, in the isothermal-isobaric (NPT) ensemble using a Berendsen barostat ${ }^{28}$ (pressure of 1 bar) and a V-rescale thermostat ${ }^{29}$ (temperature of $37^{\circ} \mathrm{C}$ ). Van der Waals interactions were truncated using a $1.2 \mathrm{~nm}$ spherical cut-off function. Electrostatic interactions were treated with the PME algorithm. The length of MD trajectories of toxin-peptide complexes and free $\alpha$-neurotoxins was $200 \mathrm{~ns}$, which is enough to sample internal conformational movements and account inter- and intramolecular contacts for this size molecular system. The length of the MD trajectories of free peptides was $500 \mathrm{~ns}$.

Computational analysis of peptides and their complexes with $\alpha$-neurotoxins. Analysis of interand intramolecular contacts in MD trajectories and interaction energy profiles evaluation was performed using an in-house software package IMPULSE [Krylov et al., in preparation] analogously to the procedures described in our previous study ${ }^{30}$.

$\mathrm{H}$-bonds were assigned using parameters set from hbond utility of GROMACS software ${ }^{25}$ (distance D-A $\leq$ $3.5 \AA$ and angle $\mathrm{D}-\mathrm{H}-\mathrm{A} \geq 150^{\circ}$ for the hydrogen bond $\mathrm{D}-\mathrm{H} \bullet \bullet \bullet \mathrm{A}$ ); salt bridges, $\pi$-cation, stacking, and hydrophobic contacts were calculated using algorithms described in our previous works ${ }^{31,32}$. All drawings showing 3D structures were prepared with the PyMOL Molecular Graphics System, version 1.8 (The PyMOL Molecular Graphics System, Version 1.8 Schrödinger, LLC.).

Gromos $9643 \mathrm{a} 3$ parameters $\mathrm{set}^{26}$ and $1.2 \mathrm{~nm}$ cutoff distance for Lennard-Jones/electrostatic interactions were used during the intermolecular short-range non-bonded interaction energy estimation. The latter being the sum of the Lennard-Jones and electrostatic terms. Graphical representation of interaction energy profiles was performed using Python built-in libraries and NumPy package.

Investigation of the molecular surface was performed using Naccess program ${ }^{33}$ and the technique described elsewhere ${ }^{34}$.

Steady-state fluorescence. The titration series for each peptide-toxin pair were performed to analyze the interaction of toxins and peptides. Each titration series was obtained by the addition of small volumes of the peptide to the stock solution of toxin, and tryptophan (Trp) fluorescence spectra at $280 \mathrm{~nm}$ excitation were obtained for each sample.

Fluorescence spectra were measured using Fluoromax-4 (Horiba Jobin Yvon). The excitation wavelength was set as $\lambda_{\text {exc }}=280 \mathrm{~nm}$ to excite proteins intrinsic fluorescence. Fluorescence emission was measured in the 300$500 \mathrm{~nm}$ wavelength region, the bandwidths of both slits were set to $2 \mathrm{~nm}$. All measurements were performed at room temperature $\left(25 \pm 1{ }^{\circ} \mathrm{C}\right)$ without temperature stabilization.

The stock solutions for fluorescence measurements were prepared using Tris- $\mathrm{HCl}$ buffer $(\mathrm{pH} 7.40 \pm 0.03$, $50 \mathrm{mM},[\mathrm{NaCl}]=0.1 \mathrm{M})$. Concentrations of stock solutions of peptides and toxins were determined spectrophotometrically using known extinction coefficients at $280 \mathrm{~nm}$. Spectra were subjected to the principal component analisys and fitted to the equations:

(1) $\mathrm{K}_{\mathrm{d}}=[\alpha$-neurotoxin $][$ binding peptide $] /[$ Complex $]$,

(2) $[\alpha \text {-neurotoxin }]_{\text {total }}=[\alpha$-neurotoxin $]+[$ Complex $]$,

(3) [binding peptide $]_{\text {total }}=[$ binding peptide $]+[$ Complex $]$,

where $\mathrm{K}_{\mathrm{d}}$ is the dissociation constant (affinity), and equilibrium concentrations of each component are shown in square brackets.

Data processing, fitting and plotting, were performed using Python programming language and NumPy, lmfit and matplotlib libraries.

Liquid chromatography. Superdex 75 10/300 GL column with AKTA Purifier (GE Healthcare Bio-Sciences, PA, USA) was utilized to determine tested peptide complex formation with the $\alpha \mathrm{Bgt}$ or $\alpha \mathrm{Ctx}$ using the following protocol: $0.1 \mathrm{M}$ ammonium acetate solution ( $\mathrm{pH} 6.2$ ), flow speed set to $0.5 \mathrm{ml} / \mathrm{min}$, optical density detection at $226 \mathrm{~nm}$ and $280 \mathrm{~nm}$. Binding reactions performed in $20 \mathrm{mM}$ Tris- $\mathrm{HCl} \mathrm{pH} 8.0$ with $30 \mu \mathrm{M} \alpha \mathrm{Bgt}$ or $\alpha \mathrm{Ctx}$ and various concentrations of HAP or HAP [L9E] peptides $(10 \mu \mathrm{M}, 20 \mu \mathrm{M}, 30 \mu \mathrm{M}, 40 \mu \mathrm{M}, 60 \mu \mathrm{M})$. Chromatography results were quantified using UNICORN Manager program. HPLC-MS analysis of fractionated peaks was performed using Thermo Finnigan LCQ Deca XP ion trap instrument with Thermo Accela UPLC system equipped with Waters Atlantis T3 $3 \mathrm{um} 150 \times 2 \mathrm{~mm}$ column. Detection was achieved by UV-VIS DAD and full scan MS (ESI+, 150-2000 au). 
A

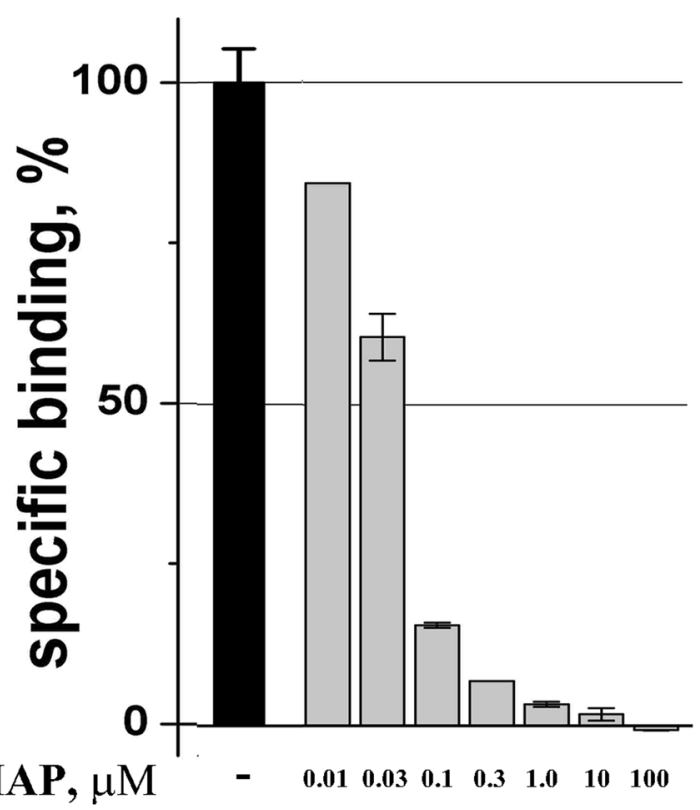

$\mathcal{C}$

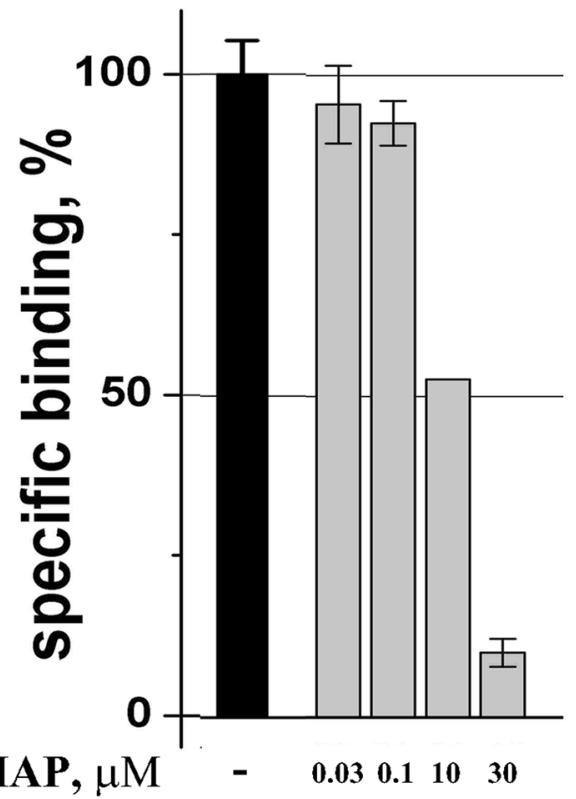

B

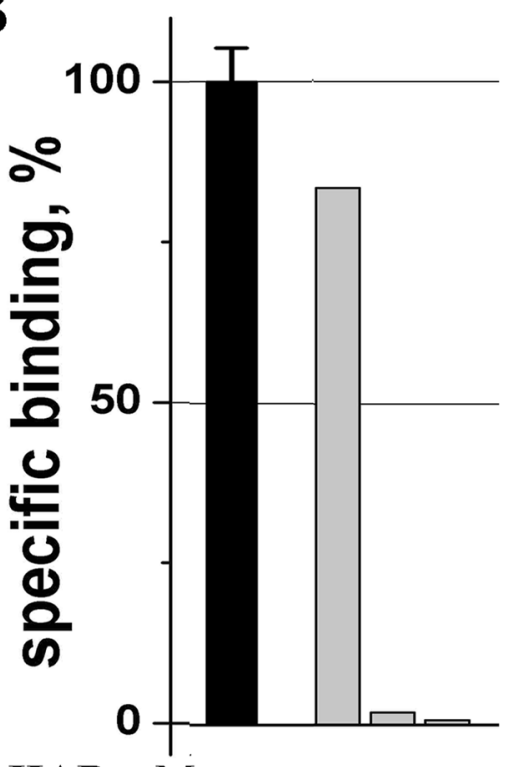

D

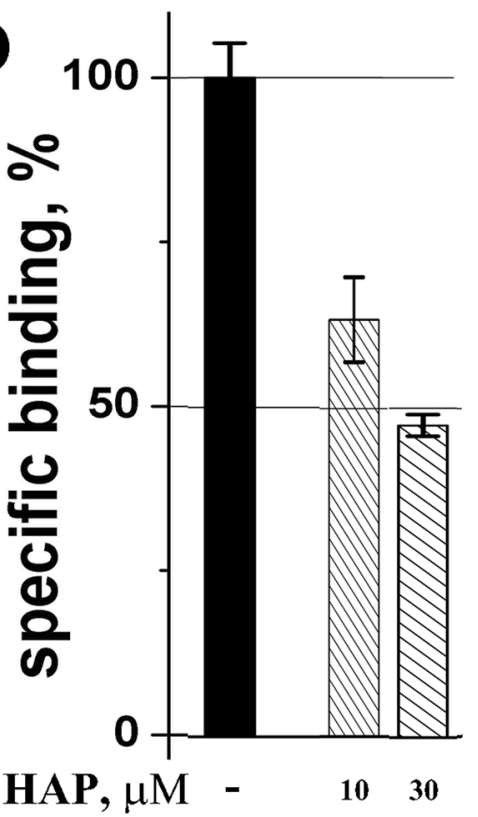

Figure 1. Verification of HAP interaction with $\alpha$ Bgt by competitive radioligand analysis. The presence of HAP diminishes dose-dependently the association of ${ }^{125} \mathrm{I}-\alpha \mathrm{Bgt}$ with all tested targets: Torpedo californica $\mathrm{nAChR}$ (A), Lymnaea stagnalis AChBP (B) and human $\alpha 7 \mathrm{nAChR}(\mathbf{C})$; however, HAP "inhibits" ${ }^{25} \mathrm{I}$ - $\alpha \mathrm{Ctx}$ binding to $T$. californica nAChR only partially at concentrations as high as $30 \mu \mathrm{M}(\mathrm{D})$.

Combined mass detection by native MS. ESI MS spectra were recorded on an LTQ Orbitrap XL instrument (Thermo Scientific, San Jose, USA) equipped with HESI-II ion source. A samples was injected with an Agilent $1100 \mu$-autosampler and isocratic pump (Agilent Technologies, Santa Clara, USA). The acquisition was performed using full-scan FTMS mode at $30 \mathrm{~K}$ resolution in a positive ions detection mode within 700-2000 mass range. The tube lens voltage was manually tuned to avoid in-source dissociation.

The selectivity of the binding was confirmed by monitoring the mixture of short $\alpha$-neurotoxin NT II from the Naja oxiana venom. Neither HAP nor HAP[L9E] showed combined mass (See supplementary figures S13 and S14).

\section{Results and Discussion}

Comparison of interactions of $\alpha \mathrm{Bgt}$ and $\alpha \mathrm{Ctx}$ with the synthetic peptides by radioligand analysis. Earlier, a high affinity of HAP for $\alpha$ Bgt was found in experiments which demonstrated that HAP pre-incubation with biotinylated $\alpha$ Bgt diminished binding of the latter to the muscle-type $\mathrm{nAChR}$ and the respective concentration dependencies provided the binding parameters. We applied radioligand analysis for the same 

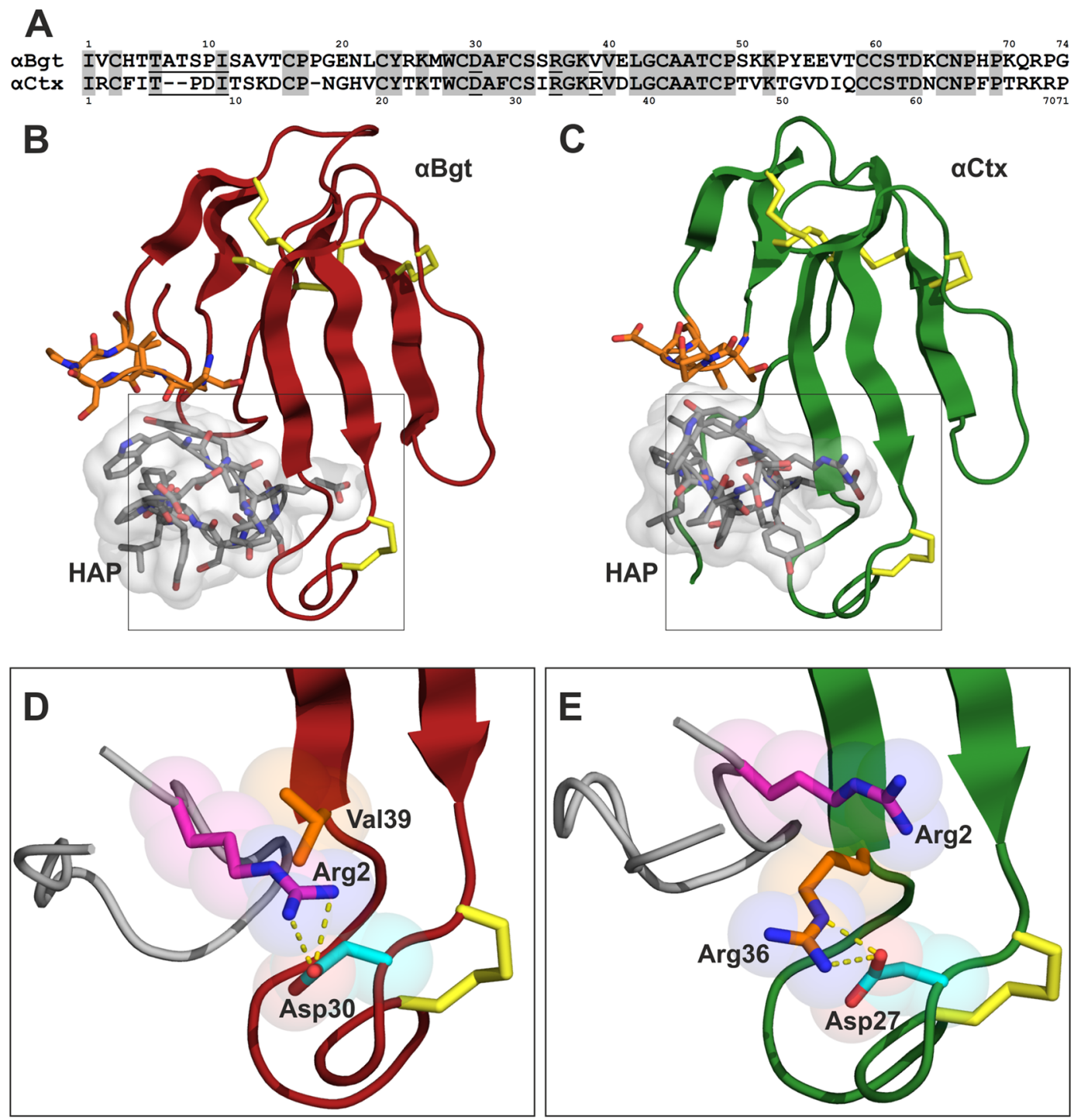

Figure 2. Analysis of the structure of the peptide-toxin complex. (A) Amino acid sequence alignment of $\alpha B g t$ and $\alpha \mathrm{Ctx}$. The numbering of the residues shown above and under the sequences. The grey background shows identical residues. Residues in the $\mathrm{N}$-terminal and central loops interacting with HAP differentially, according to intermolecular distances analysis, are underlined. (B,C) complexes of $\alpha$ Bgt and $\alpha$ Ctx with HAP after $200 \mathrm{~ns}$ of MD. $\alpha$ Bgt and $\alpha$ Ctx are shown by ribbon and coloured in red and green, respectively; HAP is shown by grey sticks and the molecular surface of HAP shown semitransparent; orange sticks show the tip of $\alpha$-neurotoxins N-terminal loop; yellow sticks show disulfide bridges. (D,E) magnified fragments inside black boxes on panels $(\mathbf{B}, \mathbf{C})$ respectively. For the clarity, $\alpha$-neurotoxins are shown partially; HAP is shown as ribbon. Pink sticks show HAP residue Arg2, $\alpha$-neurotoxins residues Asp30/Asp27 are shown by cyan sticks, orange sticks show Val39/ Arg36, nitrogen and oxygen atoms are shown in blue and red, respectively; atoms of the residues are shown as semitransparent spheres; yellow sticks show disulfide bridges; yellow dashed lines show intermolecular contacts. All drawings showing 3D structures were prepared with the PyMOL Molecular Graphics System, version 1.8 (The PyMOL Molecular Graphics System, Version 1.8 Schrödinger, LLC; https://pymol.org/2/).

purpose and compared the HAP effects on the ${ }^{125} \mathrm{I}-\alpha \mathrm{Bgt}$ binding to the muscle-type Torpedo californica and human neuronal $\alpha 7 \mathrm{nAChRs}$, as well as to Ls-AChBP. Figure 1A-C shows that in all three cases, the presence of the HAP diminished or completely suppressed the ${ }^{125} \mathrm{I}-\alpha \mathrm{Bgt}$ binding to each receptor in a dose-dependent manner. A magnitude of the effect is dependent on the $\alpha$ Bgt affinity to the tested targets. Thus, these results confirm earlier publications ${ }^{18,35}$.

In our works on nAChRs, we often used both $\alpha$ Bgt and $\alpha$ Ctx and considered them as almost identical in terms of inhibitory activity (the advantage of $\alpha \mathrm{Ctx}$ is its much higher content in the Naja kaouthia venom and the simplicity of isolation). Surprisingly, incubation of ${ }^{125} \mathrm{I}-\alpha \mathrm{Ctx}$ even with high concentrations $(30 \mu \mathrm{M})$ of HAP decreased binding of this radioactive toxin to T. californica nAChR only half (Fig. 1D), thus being indicative of a difference in the structural motifs responsible for HAP binding to these two proteins (compare Fig. 1A,D). 

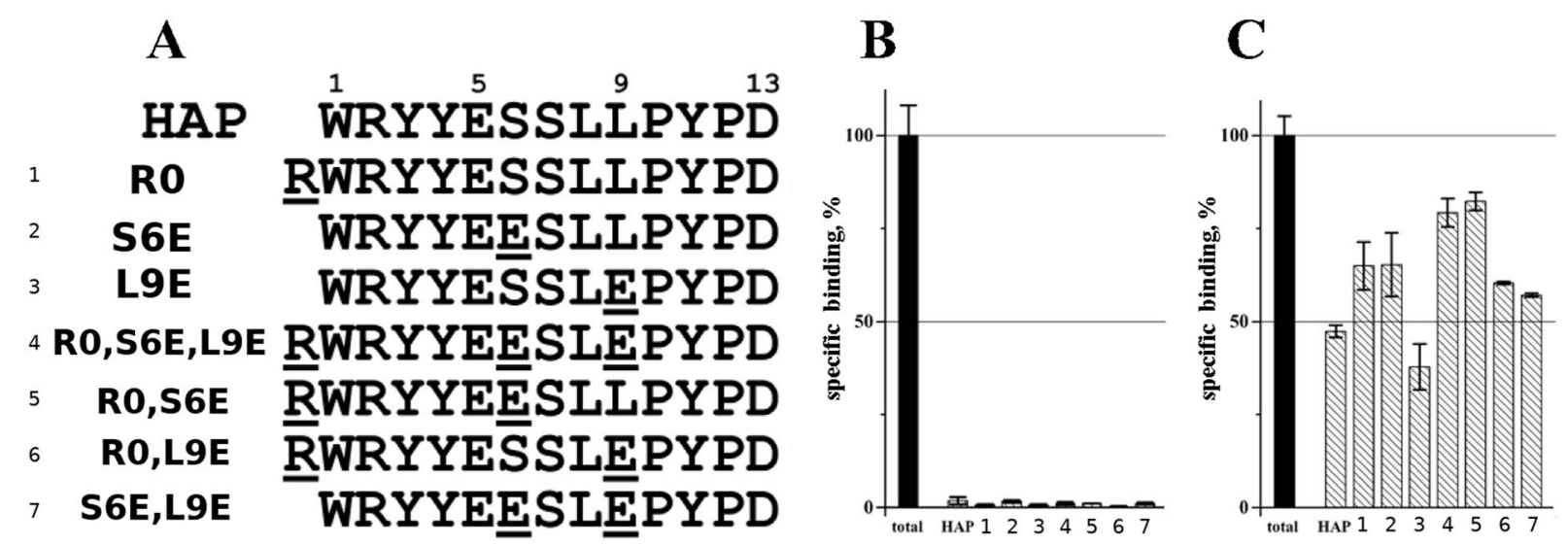

Figure 3. Rational design of the peptides capable to bind of $\alpha \mathrm{Ctx}$. (A) Amino acid sequence alignment of HAP and its point mutant variants: residues numbering is shown above the sequences. Point mutations are underlined. (B) $\left[{ }^{125} \mathrm{I}\right]-\alpha$ Bgt specific binding to T. californica $\mathrm{nAChR}$ in the presence of $10 \mu \mathrm{M} \mathrm{HAP}$ and its analogues. (C) $\left[{ }^{125} \mathrm{I}\right]-\alpha \mathrm{Ctx}$ specific binding to T. californica $\mathrm{nAChR}$ in the presence of $30 \mu \mathrm{M}$ HAP and its analogues. Total binding is measured in the absence of any peptide and expressed in terms of specific binding (\% of the general binding minus non-specific binding).

HAP showed lower apparent inhibitory activity on L. stagnalis AChBP (Fig. 1B) and especially on $\alpha 7 \mathrm{nAChR}$ in $\mathrm{GH} 4 \mathrm{C} 1$ cells (Fig. 1C) in radioligand analysis with iodinated $\alpha$-bungarotoxin. A 300 -fold increase in the concentration of HAP is required to get a similar effect with $125 \mathrm{I}-\alpha \mathrm{Ctx}$. An approximated calculation shows that a " $50 \%$ inhibition" should be observed on the L. stagnalis AChBP at $100 \mu \mathrm{M}$ concentration of HAP, and even at millimolar concentrations in the case of $\alpha 7 \mathrm{nAChR}$. Thus, such experiments were not performed.

Molecular modeling and rational design of HAP analogs. To explain such a significant difference in HAP binding to $\alpha$ Ctx and $\alpha B g t$, we carried out a computational investigation of these molecular systems. The spatial structure of the $\alpha$ Bgt-HAP complex was taken from PDB $\left(1 \mathrm{HC}^{17}\right)$. 3D model of $\alpha \mathrm{Ctx}-\mathrm{HAP}$ complex was built based on the homology with $\alpha$ Bgt-HAP complex using $\alpha$ Ctx structure derived from its complex with Ls-AChBP (PDB ID: $1 \mathrm{YI}^{24}$ ). Both complexes were subjected to $200 \mathrm{~ns}$ molecular dynamics simulation (MD). Figure 2 shows that HAP may preserve a similar orientation in both complexes. HAP forms multiple hydrophobic and specific intermolecular contacts with $\alpha$ Bgt (Supplementary Tables S1-S5), which is in good accordance with the experimental data ${ }^{17}$. However, analysis of contacts along the MD trajectories revealed significant differences in complexes $\alpha \mathrm{Bgt}-\mathrm{HAP}$ and $\alpha \mathrm{Ctx}-\mathrm{HAP}$ due to differences in the neurotoxins structure. In particular, the $\mathrm{N}$-terminal loop of $\alpha \mathrm{Ctx}$ (residues 6-9) lacks two residues as compared to the analogous loop of $\alpha \mathrm{Bgt}$ (residues 6-11) (Fig. 2A-C). For that reason, this $\alpha$ Ctx fragment forms a single H-bond with HAP, while the analogous loop of $\alpha$ Bgt forms five H-bonds (Supplementary Table S1). Besides, Arg2 of HAP forms the H-bond and salt bridge with $\alpha$ Bgt residue Asp30, while a bulky side chain of Arg36 forming intramolecular contacts with Asp27 hinders the formation of contact with homologous $\alpha$ Ctx residue Asp27 sterically (Fig. 2D,E; Supplementary Tables S1, S2).

Taking into account experimental data and efforts to design HAP as the best peptide that binds $\alpha$ Bgt with high affinity ${ }^{35}$, we tried to follow these studies using a computational approach to design a peptide that would efficiently bind $\alpha \mathrm{Ctx}$. The point mutant variants of HAP were proposed in silico based on results of MD simulation of $\alpha \mathrm{Ctx}-\mathrm{HAP}$ and $\alpha \mathrm{Bgt}-\mathrm{HAP}$ as follows. We analyzed intermolecular contacts in the complex $\alpha \mathrm{Ctx}-\mathrm{HAP}$ and taking into account what contacts formed by each residue we tried to replace some HAP residues in a way to obtain complexes where new contacts are structurally (sterically, geometrically) and physical-chemically possible. These complexes with $\alpha \mathrm{Ctx}$ (as well analogous complexes with $\alpha \mathrm{Bgt}$ ) were subjected to $200 \mathrm{~ns}$ MD simulation. Only those peptides, which demonstrated additional intermolecular contacts in the complexes with $\alpha$ Ctx and, as far as possible, did not lose contacts in the complexes with $\alpha$ Bgt were selected for the following study (Fig. 3A). The designed peptides were synthesized and tested by competitive radioligand analysis (Fig. 3B,C).

As expected, radioligand analysis confirmed that all designed HAP analogues effectively bind $\alpha$ Bgt (Fig. 3B). However, although computational analysis (that during MD suggested for each designed HAP analogue favourable intermolecular contacts with $\alpha \mathrm{Ctx}$ ), only the peptide named HAP[L9E] demonstrated stronger binding of $\alpha$ Ctx than did HAP (Fig. 3C). HAP[L9E] peptide with just a single residue change - Leu9Glu - tends to form an additional H-bond and a salt bridge with $\alpha$-neurotoxins (Fig. 4A,B, Supplementary Tables S1, S2). Notably, the HAP[L9E] complex with $\alpha$ Bgt was previously characterized by crystallography ${ }^{18}$, but there was no data in the literature on the HAP[L9E] interaction with $\alpha \mathrm{Ctx}$ - thus, it is investigated for the first time in this work.

We performed computational analysis of complex-bound HAP[L9E]/HAP amino acid residues' contributions to the interaction energy. The resulting interaction energy profile (Fig. 4C) demonstrates a significant negative energy contribution of HAP[L9E] residue Glu9, while the contribution of analogous HAP residue Leu9 is negligible. This result assumes that HAP $[\mathrm{L} 9 \mathrm{E}]$ is to bind $\alpha \mathrm{Ctx}$ with considerably higher affinity than HAP does. 
A

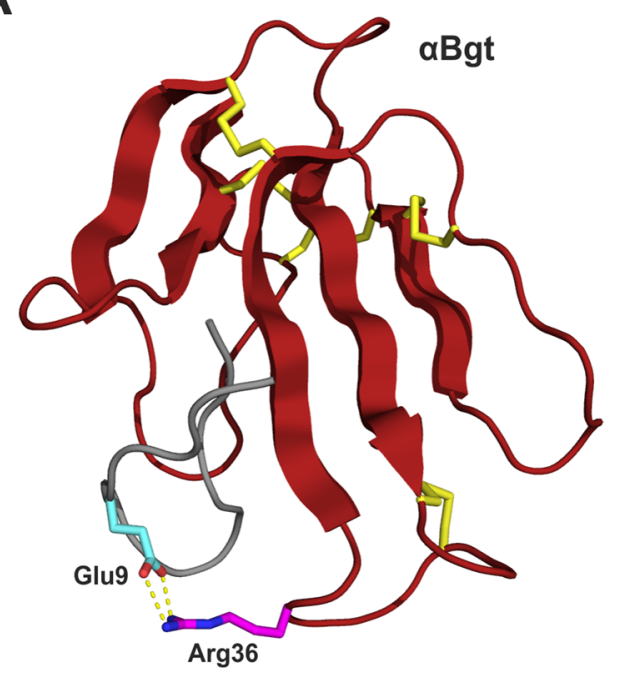

B

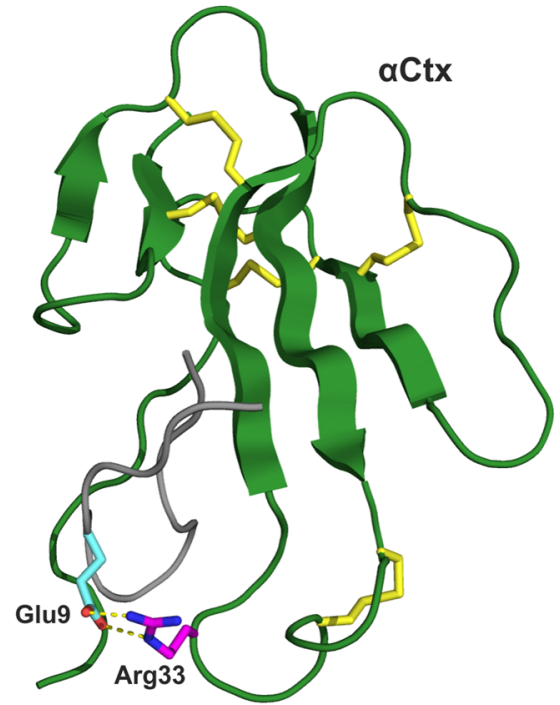

C

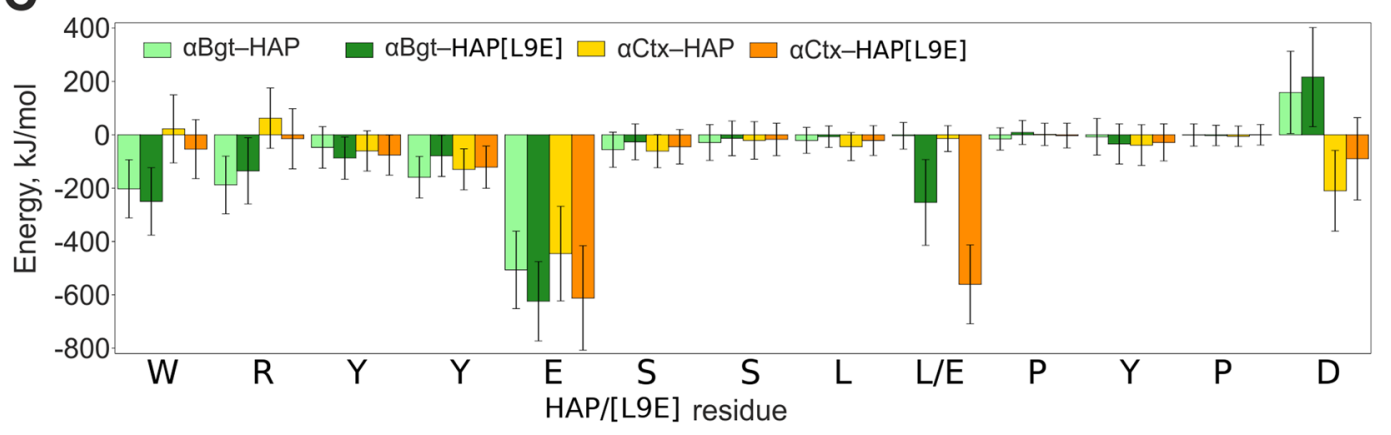

Figure 4. Molecular dynamics analisys of putative complexes between peptide HAP[L9E] (most efficiently binding $\alpha$ Ctx) and neurotoxins $\alpha$ Ctx and $\alpha$ Bgt. $(\mathbf{A}, \mathbf{B})$ complexes of $\alpha$ Bgt and $\alpha$ Ctx with HAP $[L 9 E]$ after $200 \mathrm{~ns}$ of MD. Polypeptide chains are shown as a ribbon; $\alpha$ Bgt coloured in red, $\alpha \mathrm{Ctx}-$ in green, HAP [L9E] - in grey; yellow sticks show disulfide bridges. Cyan sticks show HAP[L9E] residue Glu9; pink sticks show $\alpha$-neurotoxins residues Arg36/Arg33; nitrogen and oxygen atoms are shown in blue and red, respectively; yellow dashed lines show intermolecular contacts. (C) Interaction energy profiles of HAP/HAP [L9E] in complex with $\alpha$ Bgt and $\alpha$ Ctx. The bar chart shows amino acid residues' contributions to the interaction energy averaged over MD simulation. Error bars indicate standard deviations. Glu9 makes a positive impact (negative energy contribution), while Leu9 impact is negligible. All drawings showing 3D structures were prepared with the PyMOL Molecular Graphics System, version 1.8 (The PyMOL Molecular Graphics System, Version 1.8 Schrödinger, LLC; https://pymol.org/2/).

The results of the computational analysis suggest that peptide HAP[L9E] binds to $\alpha$ Ctx substantially more efficiently then HAP. Interestingly, most of $\mathrm{nAChR} \alpha$-subunits C-loops also contain Glu or Asp residue in the same positions. Thus, HAP[L9E] comprises a more accurate model of the receptors binding site than the original HAP. To evaluate HAP [L9E] binding affinity to $\alpha \mathrm{Ctx}$, we applied a label-free approach for measuring binding constants in HAP-toxin pairs based on intrinsic protein fluorescence spectroscopy.

\footnotetext{
A label-free fluorescence method to detect binding of HAP and its analogs to three-finger $\alpha$-neurotoxins. It should be noted that such indirect methods as monitoring of radio- or fluorescent-labelled toxin binding to the $\mathrm{nAChR}$ or GABAAR do not allow to measure constants of dissociation of studied peptides. However, these parameters are essential to rationally interpret molecular modeling and to design new HAP analogs that would be more universal at binding not only $\alpha$ Bgt but also its such homologs as $\alpha \mathrm{Ctx}$. That is why we applied an experimental approach based on the intrinsic protein fluorescence to assess binding of HAP (and its analogues) to $\alpha \mathrm{Ctx}$ and $\alpha \mathrm{Bgt}$.

Intrinsic proteins fluorescence at UV excitation is due to aromatic amino acid residues in its structure, and Trp is the major fluorophore because of its higher fluorescence quantum yield. Trp fluorescence emission properties such as intensity, fluorescence lifetime and spectral band shape are sensitive to their local microenvironment and are commonly used to study proteins' interaction. Namely, Trp fluorescence sensitivity to Trp residue mobility during the excited state lifetime, its exposure to the solvent and the polarity of the local environment allow investigation of protein conformational changes, including those caused by complex formation ${ }^{36}$.
} 

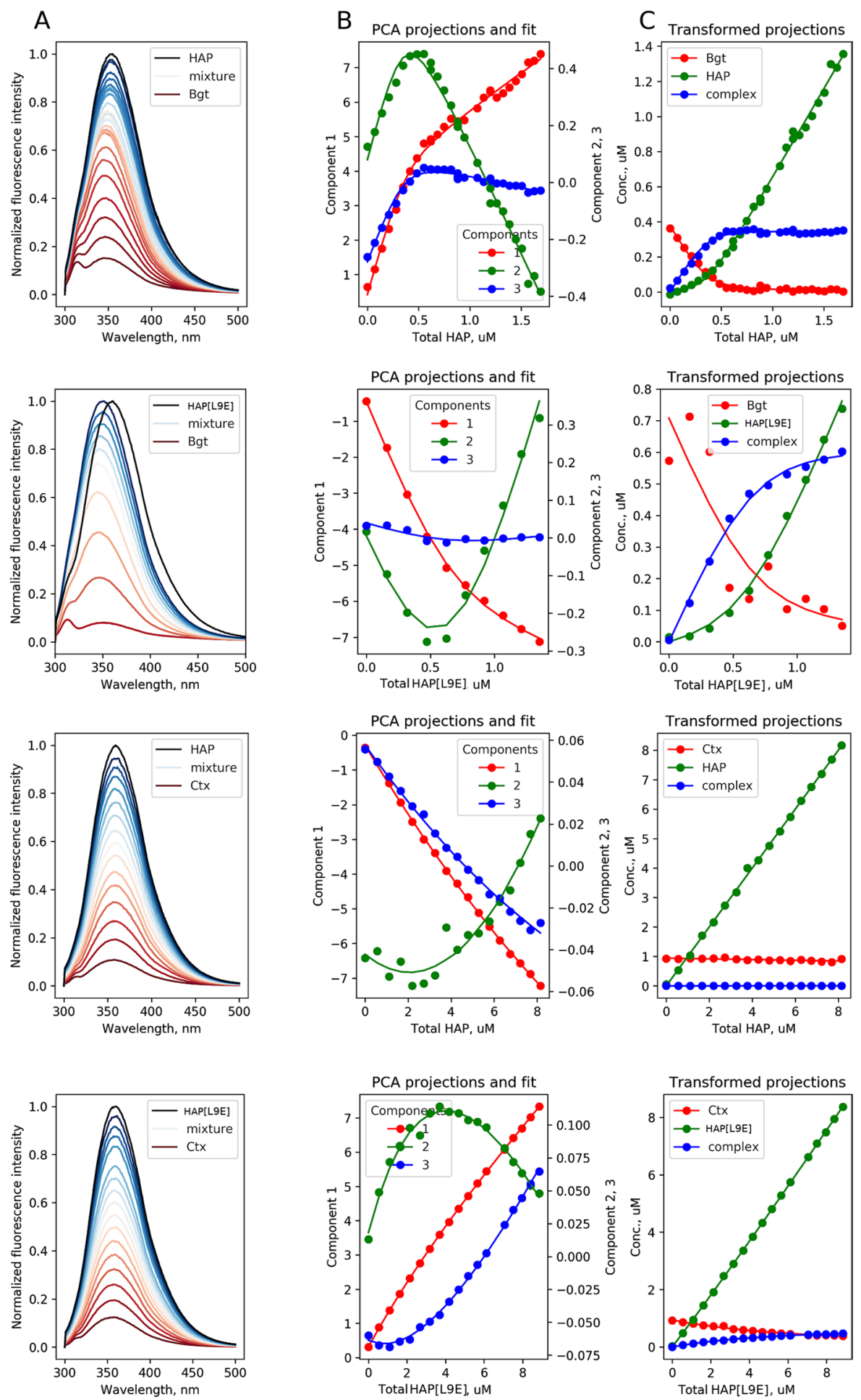

Figure 5. Fluorescence spectra and fitting results for $\alpha$ Bgt-HAP, $\alpha$ Bgt-HAP [L9E], $\alpha$ Ctx-HAP, $\alpha$ Ctx-HAP[L9E] toxin-peptide pairs. (A) Normalized fluorescence spectra of toxin (dark red), peptide (black) and their mixtures with different concentration ratios (dark red to blue). (B) Spectra projections on the first three principal components (dots) and the fit obtained using 1:1 complexation model (solid lines). The nonlinear dependence of the first projection on peptide concentration demonstrates complexation in systems, and the 1:1 model is in a good agreement with the projections obtained from the experimental data. (C) Dependence of partial concentrations of peptide, toxin and complex on total peptide concentrations obtained from PCA projections.

A blue shift of spectra was observed upon addition of peptides to $\alpha \mathrm{Bgt}$, and the dependence of fluorescence spectra maxima on peptides concentration was nonmonotonous, being indicative of binding in the system (Fig. 5). In contrast to this, the dependences of the spectra maxima position on peptides concentration for $\alpha \mathrm{Ctx}$ 


\begin{tabular}{|l|l|l|}
\hline Toxin & Peptide & $\mathbf{K}_{\mathbf{d}}, \mathbf{n M}$ \\
\hline$\alpha$ Bgt & HAP & $29 \pm 8$ \\
\hline$\alpha$ Bgt & HAP[L9E] & $91 \pm 33$ \\
\hline$\alpha C t x$ & HAP & $>10,000$ \\
\hline$\alpha C t x$ & HAP[L9E] & $7000 \pm 1000$ \\
\hline
\end{tabular}

Table 1. Dissociation constants for peptide-toxin pairs obtained from PCA+NLS fitting of fluorescence spectra.

A

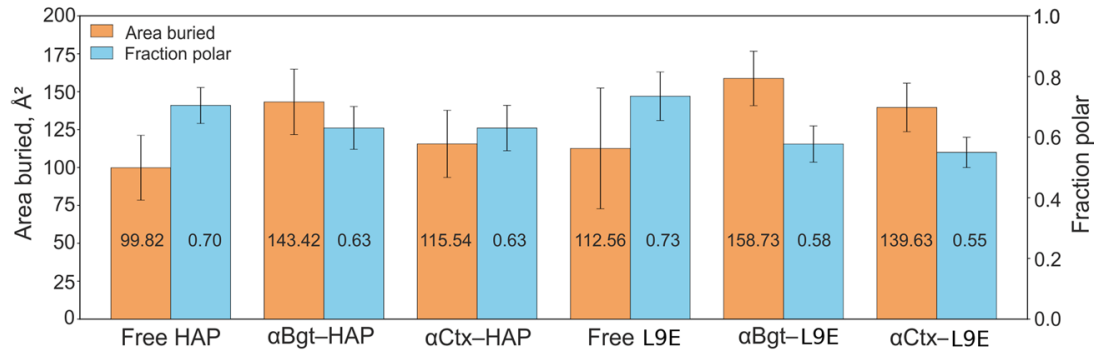

B

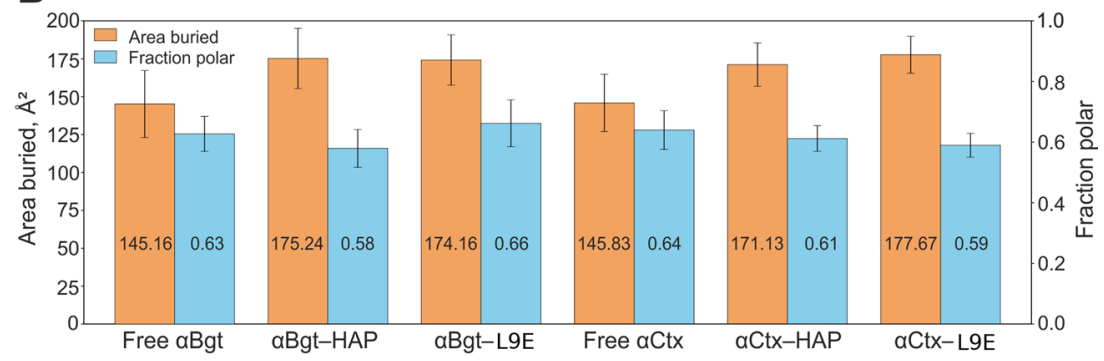

Figure 6. Area buried and fraction polar averaged over MD simulation for (A) Trp1 in free and complexbound HAP/HAP[L9E] and (B) Trp28/Trp25 in free and complex-bound $\alpha B g t / \alpha C t x$. Error bars indicate standard deviations.

were monotonous, integral fluorescence intensity dependence on peptide concentration was closer to linear, and the observed fluorescence spectra (see Fig. 5A) demonstrated only slight band shape variation. Hence, the application of the simple fitting procedure of experimental data for a single wavelength or integral intensity was complicated. So, we used the spectra processing algorithm based on the composition of principal component analysis (PCA) and nonlinear least square (NLS) fitting described in detail elsewhere ${ }^{37}$ and given as a summary with examples in Supporting information section 2. Application of PCA allowed us to make the interaction between the peptide and toxin and the arising complex formation more evident.

As can be seen from Fig. 5B, the PCA projections obtained for the system of toxin ( $\alpha \mathrm{Bgt})$ and peptide (HAP), interacting with high affinity, demonstrate a nonlinear dependence on the toxin concentration, thus providing evidence for complex formation. The dependences of the first three projections obtained from PCA on the total peptide concentration were further fitted with a $1: 1$ complex formation model $\left(\mathrm{K}_{\mathrm{d}}=[\alpha\right.$-neurotoxin] [binding peptide]/[Complex]) providing the value of dissociation constant (see in Table 1).

The applied algorithm allows transforming the projections vector into the concentration vector using the rotation matrix ( $\mathbf{P}$ in the notion of ${ }^{37}$ ) calculated using the obtained affinity constant values. Hence, one can plot concentration dependence for free peptide, toxin and their complex on the total peptide concentration (see Fig. 5C). Noteworthy, $\alpha$ Bgt demonstrated a higher affinity for both HAP and HAP[L9E] as compared to $\alpha \mathrm{Ctx}$, however, while no interaction was observed for the HAP- $\alpha$ Ctx system, it was clearly shown that the HAP[L9E] peptide binds to $\alpha \mathrm{Ctx}$ (see Fig. 5C and Table 1).

Both neurotoxins $\alpha \mathrm{Ctx}$ and $\alpha \mathrm{Bgt}$, as well as both peptides HAP and HAP [L9E], each, contain one tryptophan residue in their structure (Figs. 2A and 3A). Notably, each tryptophan residue is involved in multiple intermolecular contacts formation (Supplementary Tables S1-S5). To ascertain the fluorescence spectrum changes by the complex formation, we conducted a computational investigation of the molecular surface of each tryptophan residue in the complexes and free $\alpha \mathrm{Ctx}, \alpha \mathrm{Bgt}$, HAP and HAP[L9E]. For this purpose, we conducted an MD simulation of these polypeptide molecules in an explicitly defined water environment.

It was shown that the buried surface area ("area buried", $\mathrm{Ab}^{38}$ ) of HAP/HAP[L9E] Trp1 residue side chain in the complexes with $\alpha \mathrm{Bgt}$ is $\sim 40 / 45 \AA^{2}$ greater than in free peptides, and in the complexes with $\alpha \mathrm{Ctx}$ it is greater for $\sim 15 / 39 \AA^{2}$ (Fig. 6A). The fraction of the side chain area that is exposed to polar atoms ("fraction polar", $\mathrm{Fp}^{38}$ ) of HAP/HAP[L9E] Trp1 residue in the complexes with $\alpha$ Bgt is $~ 7 / 15 \%$ smaller than in free peptides, and in the 
A

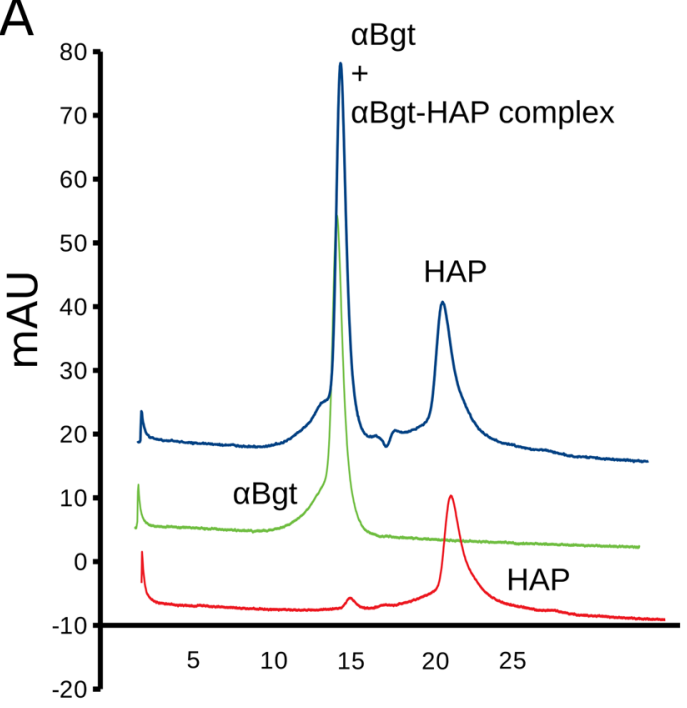

C

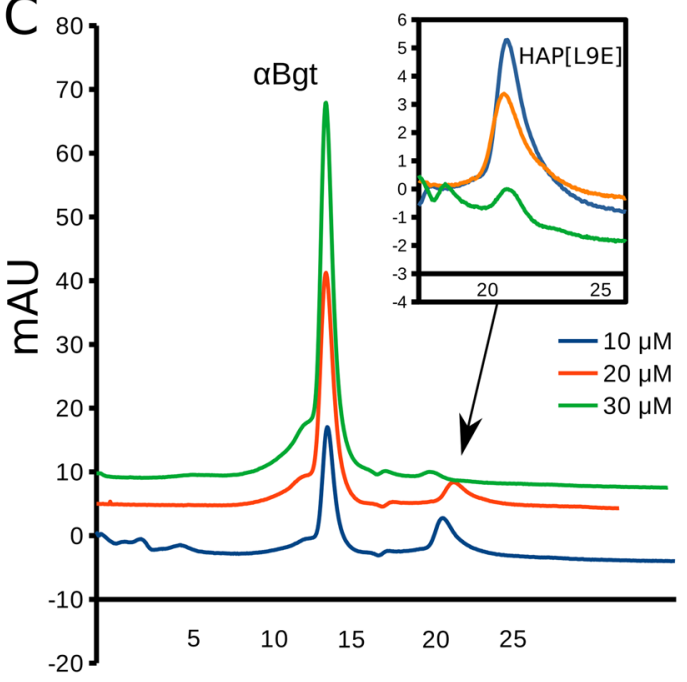

B
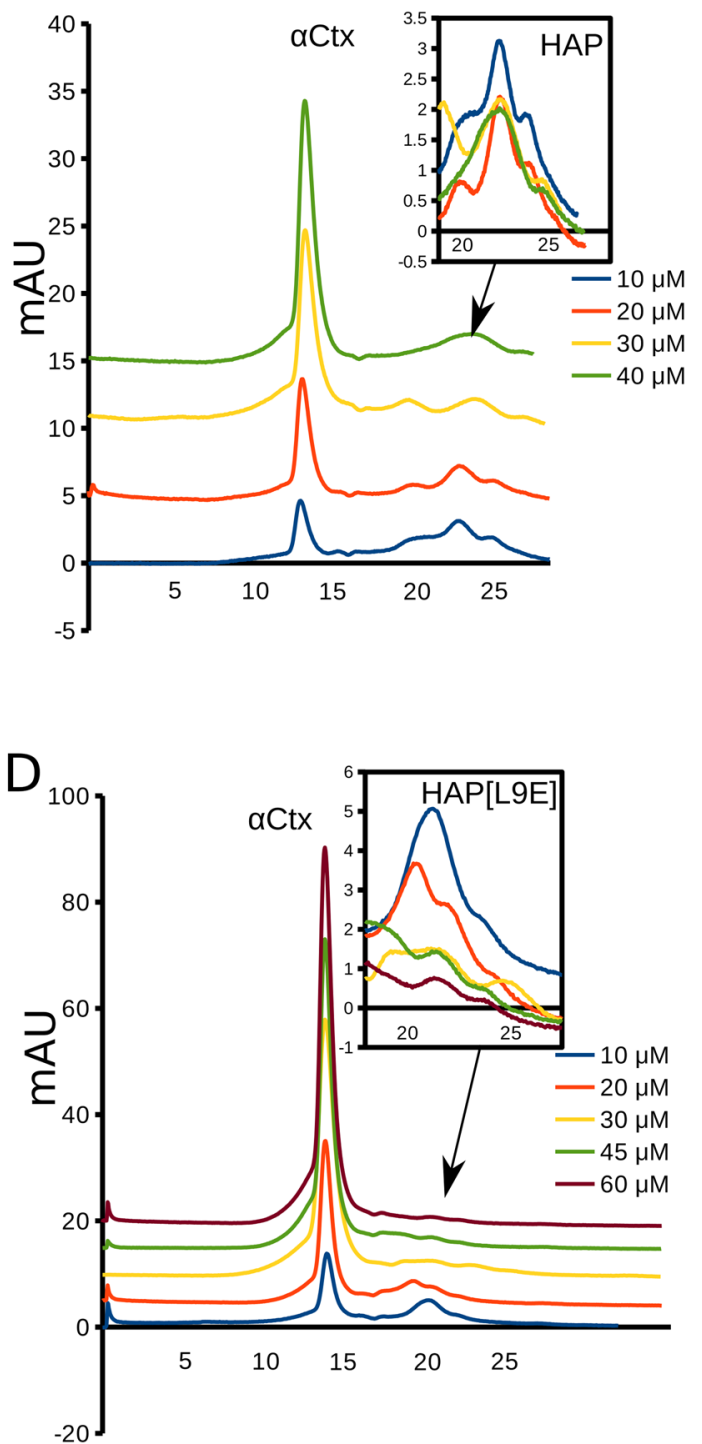

Figure 7. Monitoring the peptide-toxin complex formation via gel-filtration. (A) Gel-filtration profile of $\alpha$ Bgt (green), HAP (orange) and their mixture (blue) on the Superdex 75 10/300 GL column. Pure HAP and $\alpha$ Bgt are well separated on this column, thus allowing to monitor the formation of the complex between HAP and three-finger toxin (see Fig. S10 for the LC-MS). (B) No signs of HAP- $\alpha$ Ctx complex formation observed on the gel-filtration profile of their mixture. (C) HAP[L9E] analogue of HAP interacts with $\alpha$ Bgt in a concentrationdependent manner as can be monitored via reduction of the respective peak on gel-filtration profile with increasing concentrations of $\alpha$ Bgt. (D) HAP [L9E] analogue of HAP also interacts with $\alpha \mathrm{Ctx}$ in a concentrationdependent manner and reduction of its peak can be monitored on the gel-filtration profile with increasing concentrations of $\alpha$ Ctx.

complexes with $\alpha \mathrm{Ctx}$ it is smaller by $\sim 7 / 18 \%$. On the other hand, $\mathrm{Ab}$ of $\alpha \mathrm{Bgt} / \alpha \mathrm{Ctx} \operatorname{Trp} 28 / \operatorname{Trp} 25$ residue side chain in the complexes with HAP is $\sim 30 / 26 \AA^{2}$ greater than in free $\alpha$-neurotoxins, and in the complexes with HAP[L9E] it is greater for $\sim 29 / 32 \AA^{2}$ (Fig. 6B). Nevertheless, Fp of $\alpha B g t / \alpha C t x \operatorname{Trp} 28 / \operatorname{Trp} 25$ residues in the complexes do not differ dramatically from Fp value in free peptides: it is for $\sim 5 / 3 \%$ smaller in the complexes with HAP, and for $\sim 3 / 5 \%$ smaller in the complexes with HAP[L9E].

These results show that in HAP as well as in HAP[L9E], the Trp1 side chain becomes more buried in the complexes with the $\alpha$-neurotoxins, while the polarity of its molecular environment decreases. This observation is consistent with the results of fluorescence analysis: in case of strong Trp microenvironment influence by complexation process, the peak of the tryptophan fluorescence spectrum shifts to the shortwave region, and the dependence of spectra maxima position on peptide concentration at constant toxin concentration becomes nonmonotonous. The most pronounced variations of spectra and nonmonotonous dependence of spectra maxima correspond to $\alpha$ Bgt-HAP and $\alpha$ Bgt-HAP $[\mathrm{L} 9 \mathrm{E}]$ complexes, which are characterized by the most significant changes of Ab parameter of Trp1 of the peptide (see Fig. 6A). For $\alpha$ Ctx-HAP and $\alpha$ Ctx-HAP [L9E] pairs, the 

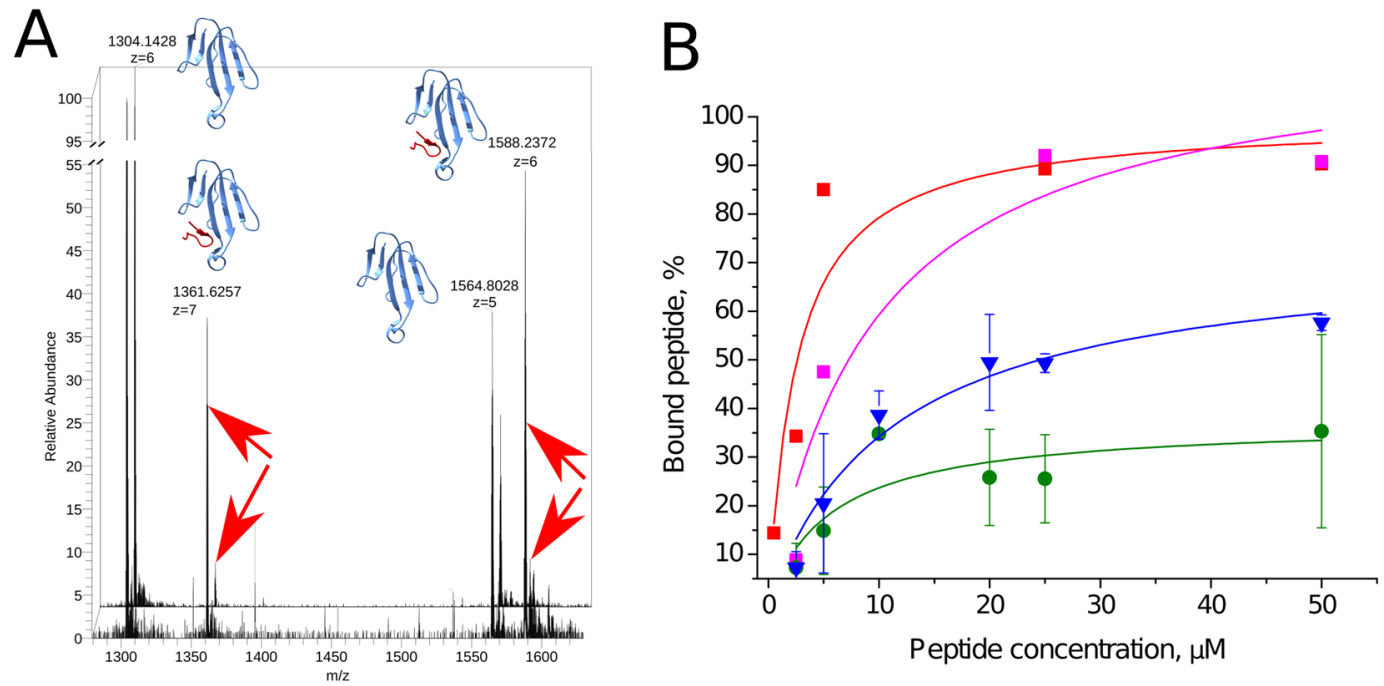

Figure 8. ESI-MS observation of the toxin and binding peptide combined mass. (A) superimposed spectra of 1:1 $\alpha$ Ctx to HAP[L9E] mixture (background spectrum) and 1:10 $\alpha$ Ctx to HAP[L9E] mixture (foreground spectrum). Note that $\mathrm{m} / \mathrm{z}$ peaks (pointed by red arrows) indicating the combined $\alpha \mathrm{Ctx}+\mathrm{HAP}[\mathrm{L} 9 \mathrm{E}]$ mass are higher in the 1:10 than in 1:1 mixture. Titration of $\alpha \mathrm{Ctx} / \alpha$ Bgt with HAP/HAP[L9E] and monitoring the intensities of the respective peaks allows the binding evaluation. (B) Titration curves fitted by the one-site binding equation: $\alpha$ Bgt and HAP (red), $\alpha$ Bgt and HAP[L9E] (magenta), $\alpha$ Ctx and HAP[L9E] (blue), $\alpha$ Ctx and HAP (green). Even though both $\alpha$ Ctx curves do not saturate at $100 \%$ (in contrast to $\alpha$ Bgt), complex HAP[L9E] saturates at a higher level $(73 \pm 3 \%)$ than HAP $(37 \pm 8 \%)$, indicating stronger binding between the toxin and mutated peptide. Drawings showing 3D structures were prepared with the UCSF Chimera, version 1.12 (http:// www.rbvi.ucsf.edu/chimera/).

variations of spectra due to complexation are less pronounced, with Trp1 Ab parameter changes due to complexation being smaller (see Fig. 6A). Vice versa, although the side chain of Trp28/Trp 25 in $\alpha$ Bgt/ $\alpha$ Ctx becomes more buried in the complexes with the peptides, it would hardly affect the fluorescence spectrum, since the polarity of Trp28/Trp25 molecular environment does not decrease much.

Validation of binding constants by gel-filtration chromatography and mass-spectrometry. Using the steady-state fluorescence measurement followed by PCA, we have detected the interaction of HAP with $\alpha$ Ctx. However, we found it necessary to validate the results of the label-free fluorescence spectroscopy by comparing them to well-established methods of direct ligand-receptor interaction measurement. To confirm that observed effects are due to the specific interaction of HAP or HAP[L9E] with $\alpha$-neurotoxins, we used gel-filtration.

Gel-filtration on the column Superdex 75 10/300 GL is capable of separating molecules in the mass range $3000-70000 \mathrm{Da}$. HAP mass is 1689 , $\alpha \mathrm{Bgt}$ and $\alpha \mathrm{Ctx}$ masses are about $7800 \mathrm{Da}$. Thus, $1: 1$ complex of HAP or its analogue with $\alpha$ Bgt or $\alpha$ Ctx should be around $9500 \mathrm{Da}$. Indeed, gel-filtration on Superdex 75 10/300 GL shows a sufficient retention time difference between HAP and $\alpha$ Bgt (Fig. 7A). However, we expected that gel-filtration would not adequately resolve HAP- $\alpha$ Bgt complex from un-bound $\alpha$ Bgt. That is why we deployed the LC-MS analysis of the first peak. Supplementary Fig. S10 shows the data from LC-MS of the first peak on HAP- $\alpha$ Bgt mixture chromatogram (blue line in Fig. 7A). Both HAP and $\alpha$ Bgt molecules detected in the first peak, showing that HAP which is significantly smaller co-elutes with $\alpha$ Bgt during gel filtration, confirming strong binding of HAP to $\alpha$ Bgt.

Figure 7B shows the gel-filtration of the series mixtures of HAP with $\alpha$ Ctx with increasing concentrations of the later. The second peak corresponding to the free HAP does not reduce upon $\alpha$ Ctx concentration increase, suggesting that complex between HAP and $\alpha \mathrm{Ctx}$ is so weak that it does not survive the gel-filtration conditions. Modified peptide HAP[L9E], on the contrary, shows strong complexation with both $\alpha$ Bgt (Fig. 7C) and $\alpha \mathrm{Ctx}$ (Fig. 7D). We performed several co-elutions for each pair: HAP[L9E]- $\alpha$ Bgt and HAP[L9E]- $\alpha$ Ctx using increasing concentrations of the particular toxins. Higher concentrations of the toxins bind more considerable amount of the HAP[L9E] peptide which leads to a gradual decrease of the second peak on the gel-filtration elution profile because higher quantities of the peptide elute in the first peaks. Therefore, HAP[L9E] peptide, which exhibited binding to both $\alpha \mathrm{Ctx}$ and $\alpha \mathrm{Bgt}$ in fluorescence measurements, showed a concentration-dependent interaction (monitored as free HAP[L9E] peak area reduction) with both $\alpha$ Bgt (Fig. 7C) and $\alpha$ Ctx (Fig. 7D), thus confirming our observations from PCA of Trp fluorescence spectra and molecular modeling. LC-MS confirmed co-elution of HAP[L9E] with both $\alpha$ Bgt and $\alpha$ Ctx in the first peaks (Figs. S11-S12).

Co-elution in the gel-filtratinon confirms that HAP[L9E] binds the $\alpha$ Ctx. But how strong and how specific this interaction might be? To investigate the interaction in more detail we performed electrospray ionisation mass-spectrometry (ESI-MS) with Orbitrap detection of the non-covalent complexes of toxins and binding peptides (Fig. 8). The principles of this method was described by Tjernberg et al. ${ }^{39}$. 
Indeed, we observe the combined $\mathrm{m} / \mathrm{z}$ on the HAP $[\mathrm{L} 9 \mathrm{E}]$ and $\alpha \mathrm{Ctx}$ mixture spectra (Fig. 8A). And its intensity depends on the HAP [L9E] concentration, complementing the gel-filtration experiment. Titration of $\alpha \mathrm{Ctx} / \alpha \mathrm{Bgt}$ with HAP/HAP [L9E] and monitoring the intensities of the respective peaks followed by fitting the dependency to one-site competition equation (Fig. $8 \mathrm{~B}$ ) allowed us to prove the complexation of HAP[L9E] with $\alpha$ Ctx directly. We also detected $\alpha$ Ctx complexation with HAP, which confirms the radioligand binding data (Fig. 3C). It should be noted that $\alpha$ Ctx seems to be not fully saturated neither by HAP nor HAP [L9E] which did not allow us to estimate binding constants. This observation confirms that the complexes with $\alpha \mathrm{Ctx}$ are weaker than the respective complexes with $\alpha$ Bgt and partially degrade during electrospray ionisation. However, HAP[L9E] curve shows a higher saturation level which confirms that the $\alpha \mathrm{Ctx}-\mathrm{HAP}[\mathrm{L} 9 \mathrm{E}]$ complex is more stable than $\alpha \mathrm{Ctx}-\mathrm{HAP}$.

\section{Conclusions}

Here, using competitive radioligand analysis, intrinsic protein fluorescence spectroscopy with PCA-based processing, gel-filtration and mass-spectrometry, we confirmed the earlier finding of Harel et al. that a 13-membered peptide HAP has an extremely high affinity for $\alpha$ Bgt. Unexpectedly, we detected only weak binding of HAP to $\alpha \mathrm{Ctx}$. Thus, $\alpha \mathrm{Bgt}$ and $\alpha \mathrm{Ctx}$, known to be very similar in their affinity for $\mathrm{nAChRs,} \mathrm{differ} \mathrm{in} \mathrm{this} \mathrm{characteris-}$ tic towards AChBPs, GABAA receptors and, as shown here, to such C-loop analogue as HAP. With molecular dynamics, we deduced the molecular properties of the affinity peptides complexes and suggested the way to improve affinity toward $\alpha \mathrm{Ctx}$. Using radioligand competition method, we selected the most effective $\alpha \mathrm{Ctx}$ binding peptide. To measure the binding constants label-free, we acquired intrinsic Trp fluorescence measurement with spectra principal component analysis. Information found in this article could be used in the design of experiments involving HAP-based tags and fluorescent derivatives of $\alpha \mathrm{Bgt}$ and $\alpha \mathrm{Ctx}$.

Received: 20 August 2019; Accepted: 14 February 2020;

Published online: 02 March 2020

\section{References}

1. Tsetlin, V. I. Three-finger snake neurotoxins and Ly6 proteins targeting nicotinic acetylcholine receptors: pharmacological tools and endogenous modulators. Trends Pharmacol. Sci. 36, 109-23 (2015).

2. Kini, R. M. \& Doley, R. Structure, function and evolution of three-finger toxins: mini proteins with multiple targets. Toxicon 56, 855-67 (2010).

3. Utkin, Y. N. Three-finger toxins, a deadly weapon of elapid venom - Milestones of discovery. Toxicon 62, 50-55 (2013).

4. Tsetlin, V., Utkin, Y. \& Kasheverov, I. Polypeptide and peptide toxins, magnifying lenses for binding sites in nicotinic acetylcholine receptors. Biochem. Pharmacol. 78, 720-31 (2009).

5. Del Bufalo, A., Cesario, A., Salinaro, G., Fini, M. \& Russo, P. Alpha9Alpha10 Nicotinic Acetylcholine Receptors as Target for the Treatment of Chronic Pain | BenthamScience. Curr. Pharm. Des. 20, 6042-7 (2014).

6. Elgoyhen, A. B. et al. alpha10: a determinant of nicotinic cholinergic receptor function in mammalian vestibular and cochlear mechanosensory hair cells. Proc. Natl. Acad. Sci. USA 98, 3501-6 (2001).

7. Dubovskii, P. V., Konshina, A. G. \& Efremov, R. G. Cobra cardiotoxins: membrane interactions and pharmacological potential. Curr. Med. Chem. 21, 270-87 (2013).

8. Vasilyeva, N. A., Loktyushov, E. V., Bychkov, M. L., Shenkarev, Z. O. \& Lyukmanova, E. N. Three-Finger Proteins from the Ly6/uPAR Family: Functional Diversity within One Structural Motif. Biochemistry. (Mosc). 82, 1702-1715 (2017).

9. Miwa, J. M., Anderson, K. R. \& Hoffman, K. Lynx Prototoxins: The Roles of Endogenous Mammalian Neurotoxin-like Proteins in Modulating nAChR Function and Complex Nicotinic-Dependent Processes. Front. Pharmacol. 10, 343 (2019).

10. Smit, A. B. et al. A glia-derived acetylcholine-binding protein that modulates synaptic transmission. Nature 411, 261-8 (2001).

11. Brejc, K. et al. Crystal structure of an ACh-binding protein reveals the ligand-binding domain of nicotinic receptors. Nature 411, 269-76 (2001).

12. Rucktooa, P., Smit, A. B. \& Sixma, T. K. Insight in nAChR subtype selectivity from AChBP crystal structures. Biochem. Pharmacol. 78, 777-87 (2009).

13. Zouridakis, M. et al. Crystal structures of free and antagonist-bound states of human $\alpha 9$ nicotinic receptor extracellular domain. Nat. Struct. Mol. Biol. 21, 976-980 (2014).

14. Zouridakis, M. et al. Crystal Structure of the Monomeric Extracellular Domain of $\alpha 9$ Nicotinic Receptor Subunit in Complex With $\alpha$-Conotoxin RgIA: Molecular Dynamics Insights Into RgIA Binding to $\alpha 9 \alpha 10$ Nicotinic Receptors. Front. Pharmacol. 10, 474 (2019).

15. Scarselli, M. et al. NMR structure of alpha-bungarotoxin free and bound to a mimotope of the nicotinic acetylcholine receptor. Biochemistry 41, 1457-63 (2002).

16. Hannan, S., Wilkins, M. E., Thomas, P. \& Smart, T. G. Tracking Cell Surface Mobility of GPCRs Using $\alpha$-Bungarotoxin-Linked Fluorophores. in. Methods in enzymology 521, 109-129 (2013).

17. Harel, M. et al. The binding site of acetylcholine receptor as visualized in the X-ray structure of a complex between $\alpha$-bungarotoxin and a mimotope peptide. Neuron 32, 265-75 (2001).

18. Scherf, T. et al. A $\beta$-hairpin structure in a 13-mer peptide that binds $\alpha$-bungarotoxin with high affinity and neutralizes its toxicity. Proc. Natl. Acad. Sci. 98, 6629-6634 (2001).

19. Slagboom, J. et al. Neurotoxicity fingerprinting of venoms using on-line microfluidic AChBP profiling. Toxicon 148, 213-222 (2018).

20. Albulescu, L.-O. et al. A Decoy-Receptor Approach Using Nicotinic Acetylcholine Receptor Mimics Reveals Their Potential as Novel Therapeutics Against Neurotoxic Snakebite. Front. Pharmacol. 10 (2019).

21. Kryukova, E. V. et al. From Synthetic Fragments of Endogenous Three-Finger Proteins to Potential Drugs. Front. Pharmacol. 10 (2019).

22. Kasheverov, I. E. et al. High-Affinity $\alpha$-Conotoxin PnIA Analogs Designed on the Basis of the Protein Surface Topography Method. Sci. Rep. 6 (2016)

23. Kasheverov, I. E. et al. Scorpion toxins interact with nicotinic acetylcholine receptors. FEBS Lett. 593 (2019).

24. Bourne, Y., Talley, T. T., Hansen, S. B., Taylor, P. \& Marchot, P. Crystal structure of a Cbtx-AChBP complex reveals essential interactions between snake alpha-neurotoxins and nicotinic receptors. EMBO J. 24, 1512-22 (2005).

25. Abraham, M. J. et al. Gromacs: High performance molecular simulations through multi-level parallelism from laptops to supercomputers. SoftwareX, https://doi.org/10.1016/j.softx.2015.06.001 (2015).

26. Schuler, L. D., Daura, X. \& Van Gunsteren, W. F. An improved GROMOS96 force field for aliphatic hydrocarbons in the condensed phase. J. Comput. Chem., https://doi.org/10.1002/jcc.1078 (2001). 
27. Berendsen, H. J. C., Postma, J. P. M., van Gunsteren, W. F. \& Hermans, J. Interaction Models for Water in Relation to Protein Hydration. in, https://doi.org/10.1007/978-94-015-7658-1_21 (1981).

28. Berendsen, H. J. C., Postma, J. P. M., Van Gunsteren, W. F., Dinola, A. \& Haak, J. R. Molecular dynamics with coupling to an external bath. J. Chem. Phys., https://doi.org/10.1063/1.448118 (1984).

29. Bussi, G., Donadio, D. \& Parrinello, M. Canonical sampling through velocity rescaling. J. Chem. Phys., https://doi. org/10.1063/1.2408420 (2007).

30. Berkut, A. A. et al. Protein Surface Topography as a tool to enhance the selective activity of a potassium channel blocker. J. Biol. Chem. jbc.RA119.010494, https://doi.org/10.1074/jbc.ra119.010494 (2019).

31. Pyrkov, T. V. \& Efremov, R. G. A fragment-based scoring function to re-rank ATP docking results. Int. J. Mol. Sci., https://doi. org/10.3390/i8111083 (2007).

32. Pyrkov, T. V., Chugunov, A. O., Krylov, N. A., Nolde, D. E. \& Efremov, R. G. PLATINUM: A web tool for analysis of hydrophobic/ hydrophilic organization of biomolecular complexes. Bioinformatics, https://doi.org/10.1093/bioinformatics/btp111 (2009).

33. Hubbard, S. J. \& Thornton, J. M. Naccess. Comput. Progr. (1993).

34. Lüthy, R., Bowie, J. U. \& Eisenberg, D. Assessment of protein models with three-dimensional profiles. Nature, https://doi. org/10.1038/356083a0 (1992).

35. Kasher, R. et al. Design and synthesis of peptides that bind $\alpha$-bungarotoxin with high affinity. Chem. Biol., https://doi.org/10.1016/ S1074-5521(00)90063-2 (2001).

36. Lakowicz, J. R. Principles of Fluorescence Spectroscopy, https://doi.org/10.1007/978-0-387-46312-4 (Springer, Boston, MA, 2006).

37. Al-Soufi, W., Novo, M. \& Mosquera, M. Principal Component Global Analysis of Fluorescence and Absorption Spectra of 2-(2'-Hydroxyphenyl)Benzimidazole. Appl. Spectrosc. 55, 630-636 (2001).

38. Bowie, J. U., Lüthy, R. \& Eisenberg, D. A method to identify protein sequences that fold into a known three-dimensional structure. Science, https://doi.org/10.1126/science.1853201 (1991).

39. Tjernberg, A. et al. Determination of dissociation constants for protein-ligand complexes by electrospray ionization mass spectrometry. Anal. Chem., https://doi.org/10.1021/ac0497914 (2004).

\section{Acknowledgements}

The molecular dynamics simulations were carried out using the computational facilities of the Supercomputer Center "Polytechnical" at the St. Petersburg Polytechnic University and IACP FEB RAS Shared Resource Center "Far Eastern Computing Resource" equipment (https://cc.dvo.ru). We thank Eli Lilly (UK) for GH4C1 cells transfected with human $\alpha 7 \mathrm{nAChR}$ and Prof. Dr. Ferdinand Hucho (Free University of Berlin, Berlin, Germany) for T. californica electric organ preparation. Peptide synthesis and molecular modeling was supported by RFBR grant \#17-34-80084 to V.M.T.; I.E.K. was supported by the RFBR grant \#18-04-01366; V.I.T. was supported by RSF grant \#16-14-00215p, label-free analysis (gel-filtration and mass-spectrometry experiments) was supported by RSF grant \#18-74-10088 to D.S.K., I.V.S., A.V.E. and I.A.I.

\section{Author contributions}

V.I.T. designed the project concept and wrote the paper, I.V.S. and E.A.S. supervised the work and wrote the article, N.S.E. and M.N.Z. synthetized HAP and its analogues, I.E.K. and E.V.K. performed the radioligand analysis, V.M.T. and R.G.E. performed molecular modelling, MD simulations, computation of residues' contributions to the interaction energy and computational analysis, N.G.Z. measured the fluorescence spectra, G.S.B. performed the principal component analysis of the spectra, D.S.K. designed, performed and partially analyzed gel-filtration and mass-spectrometry experiments, S.Y.B. performed gel-filtration and analyzed the results, I.A.I. and A.V.J. performed mass-spectrometry. All authors contributed to the preparation of the manuscript and figures.

\section{Competing interests}

The authors declare no competing interests.

\section{Additional information}

Supplementary information is available for this paper at https://doi.org/10.1038/s41598-020-60768-y.

Correspondence and requests for materials should be addressed to V.I.T.

Reprints and permissions information is available at www.nature.com/reprints.

Publisher's note Springer Nature remains neutral with regard to jurisdictional claims in published maps and institutional affiliations.

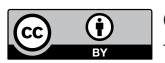

Open Access This article is licensed under a Creative Commons Attribution 4.0 International License, which permits use, sharing, adaptation, distribution and reproduction in any medium or format, as long as you give appropriate credit to the original author(s) and the source, provide a link to the Creative Commons license, and indicate if changes were made. The images or other third party material in this article are included in the article's Creative Commons license, unless indicated otherwise in a credit line to the material. If material is not included in the article's Creative Commons license and your intended use is not permitted by statutory regulation or exceeds the permitted use, you will need to obtain permission directly from the copyright holder. To view a copy of this license, visit http://creativecommons.org/licenses/by/4.0/.

(C) The Author(s) 2020 